
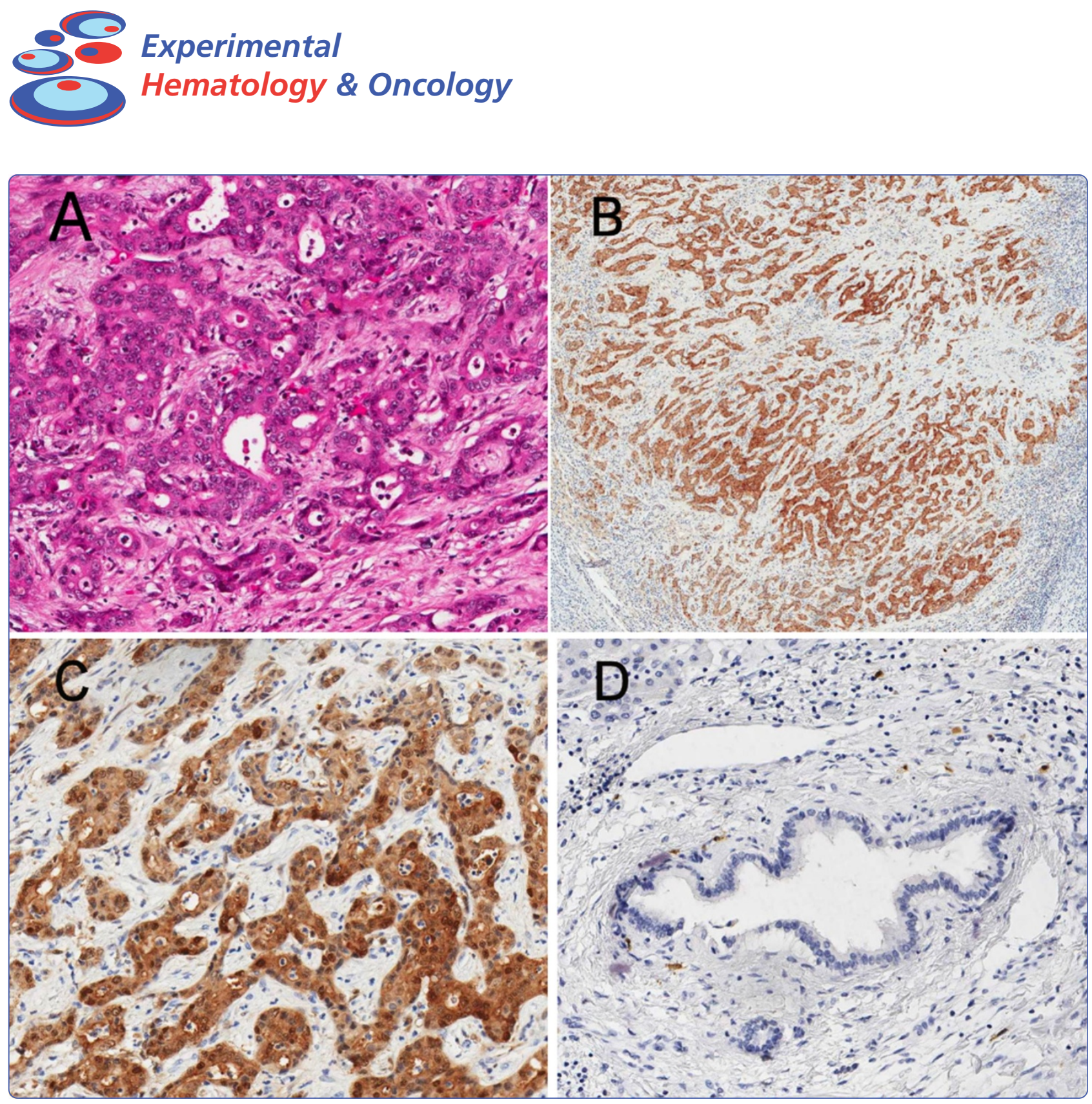

\title{
Expression and diagnostic values of calretinin and CK5/6 in cholangiocarcinoma
}

Zhang et al. 


\title{
Expression and diagnostic values of calretinin and CK5/6 in cholangiocarcinoma
}

\author{
Lanjing Zhang ${ }^{1,2,3}$, Renee Frank', Emma E Furth', Amy F Ziober', Virginia A LiVolsi ${ }^{1}$ and Paul J Zhang ${ }^{1,4^{*}}$
}

\begin{abstract}
Background: Mesothelin, a mesothelial marker, has been found expressed in and as a potential treatment target of cholangioacarcinoma (CC). It is possible that CC may be derived from the cells sharing mesothelial markers. However, the expression of other mesothelial markers in CC is largely unknown.

Methods: Thirty CC cases (10 extrahepatic and 20 intrahepatic) were retrieved from our institutional archive. The immunohistochemical study of Calretinin (DC8), WT1 (6F-H2), Lymphatic Endothelial Marker (D2-40), CK5/6 (D5/16 B4) and CK19 (b170) was done on formalin fixed paraffin embedded sections for 2-3 blocks of each case. We compared the expression levels between CC and normal bile duct (NBD) on the same block.

Results: All of the CC and NBD are positive for CK19 (23/23) and negative for WT1 (0/23) and D2-40 (0/23), except one CC positive for D2-40(1/30, 3.3\%) and one NBD positive for WT1 (1/23, 4.3\%). Calretinin immunoreactivity was detected in 52.2\% (12/23) of CC, but none in NBD (0/23). CK5/6 was also detectable in 73.3\% (22/30) of CC and all NBD (30/30). Increased expression of calretinin and reduced expression of CK5/6 were more likely associated with $C C$ than NBD $(P<0.001$ and $P=0.002$, respectively). The sequential staining pattern of positive calretinin and negative CK5/6 in calretinin negative cases has a sensitivity of $69.57 \%$ and a specificity of $100 \%$ for differentiating CC from NBD. CK5/6 expression was also more likely associated with well-differentiated CC (7/7 versus 12/20 in moderately differentiated, and $9 / 10$ in poorly differentiated, $P=0.019)$ and extrahepatic CC (10/10 versus 12/20 in intrahepatic, $P=0.029$ ), but there was no association between the calretinin expression and the CC grade or location.

Conclusion: Calretinin and CK5/6 immunohistochemical stains may be useful for diagnosing a CC. Their immunohistochemical results should be interpreted with caution in the cases with differential diagnoses of mesothelioma and CC. A full mesothelioma panel, including WT1 and/or D2-40, is recommended to better define a mesothelial lineage. The biology of calretinin and CK5/6 expression in CC is unclear, but might shed light on identifying therapeutic targets for CC.
\end{abstract}

Keywords: Cholangiocarcinoma, Calretinin, CK5/6 and immunohistochemistry, Differentiation

\section{Introduction}

Intrahepatic cholangiocarcinoma $(\mathrm{CC})$ is a relatively rare carcinoma of the biliary tree, with rising incidence and mortality [1,2]. Its 1-, 2- and 5-year survivals in US are $24.5 \%, 11.8 \%$ and $3.2 \%$, respectively [1]. The diagnosis and prognostication of $\mathrm{CC}$ become critical for managing those patients. Studies have shown that several immunohistochemical (IHC) markers are highly expressed in CC

\footnotetext{
*Correspondence: pjz@mail.med.upenn.edu

1 Department of Pathology and Laboratory Medicine, University of Pennsylvania Pearlman School of Medicine, Philadelphia, PA, USA ${ }^{4}$ Department of Pathology, 6 Founders, 3400 Spruce St, Philadelphia, PA 19104, USA

Full list of author information is available at the end of the article
}

including Annexin A1 (94.1\%), CK19 (89\%), MOC31 (88.2\%), CK7 (83.4\%), CD133 (79\%), claudin4 (69.2\%), high mobility group A1 (HMGA1) (31.5\%) and S100P [3-6], while others has no or very low expression in CC such as glypican 3 (GPC3) (7\%) and biglycan (7\%) [3]. However, the markers' expression levels are rarely compared with that of normal bile duct (NBD) which could be a morphologic mimic for $\mathrm{CC}$ in small lesion or small sampling. In addition to pancreatic carcinoma, a recent study shows that mesothelin, a mesothelial marker, is also found in $33 \%$ of resected CC specimens, but not hepatocellular carcinoma $(\mathrm{HCC})$ or normal liver tissue [7]. Moreover, mesothelin may be used as a target for 
monoclonal antibody therapy in a subset of CC in mice and as a prognostic factor for $\mathrm{CC}[8,9]$. It is possible that mesothelium related proteins and/or genes may also be present in other tumors and involved in their tumorigenesis. Indeed, mesothelin and calretinin are found expressed in thymic carcinoma, thymoma, and non-keratinizing squamous cell carcinoma of lung $[10,11]$. But little is known regarding the expression levels, if any, of other mesothelial markers such as calretinin, CK5/6, D2-40 and WT1. Hence, we aimed to examine the IHC staining pattern of those markers in CC and NBD, and to explore the potential "mesothelial" phenotype in $\mathrm{CC}$. The findings may help identify more diagnostic markers and therapeutic targets for $\mathrm{CC}$.

\section{Materials and methods}

Histologically and clinically well documented CC cases were identified and retrieved from our institutional archive. The inclusion criteria included: 1. diagnosis of cholangiocarcinoma could be confirmed by review of the slides; 2. A primary tumor must clinically and pathologically arise within the hepatobiliary system, 3. patient had no past and current history of tumors in other system, 4. It was a resection specimen and had at least 3 blocks with carcinoma available for immunohistochemical (IHC) stains; 5 . It had both CC and nearby NBD present on the same slide (A control H\&E slide was made to confirm this, after being cut for IHC stains).

The IHC protocol and related antibodies have been described before [12]. Briefly, the IHC stains of M2A antigen (clone D2-40, 1:25, Signet Laboratories, Dedham, MA), WT1 (clone 6F-H2, 1:400, DakoCytomation, Carpinteria, CA), calretinin (DC8, 1:50, Zymed Laboratories, South San Francisco, CA), and cytokeratin 5/6 (clone D5/16 B4, 1:25, DakoCytomation) and CK19 (clone b170, 1:100, Leica/Novocastra) were conducted on formalin fixed paraffin embedded tissue sections with standard IHC protocols (BondMax, Leica Microsystem, Buffalo Grove, IL) on 2-3 blocks of each case. Appropriate positive and negative controls were performed and validated.

The protein expression levels were independently assessed by two of the authors (LZ and RF). When a disagreement was present, the two would have a discussion, consult with the senior author (PJZ) and reach an agreement upon re-review of the case. A scoring scale of 0-3 was used, with 0 for negative, $1+$ for $<25 \%, 2+$ for 26 $75 \%$, and $3+$ for $>75 \%$. The staining intensity was not considered for the purpose of scoring the stains. The literature search for related IHC markers' positive rates was performed by using the marker's names and tumors as the search term in Pubmed (NCBI, NIH, USA) in Feb. 2014.
The statistical analyses were performed by using Stata (version 11, StataCorp LP). The 95\% confidence intervals (CI) were calculated by using normal distribution. Pearson Chi-square test was used to determine the association between IHC markers and tissue samples, so was Fisher exact test as a confirmation for groups with case number fewer than 6 . All of the $P$ values were calculated for 2 -sided. A $P$ value less than $5 \%$ was considered statistically significant.

\section{Results}

A total of $30 \mathrm{CC}$ cases (10 extrahepatic and 20 intrahepatic) with nearby NBD met the inclusion criteria and were included in this retrospective study. They were collected between 2005 and 2011. The stained slides from the 2 to 3 blocks of each case all showed similar IHC staining pattern for each marker evaluated. The IHC stains for D2-40 and CK5/6 were performed on all of the 30 cases, and for calretinin, CK19 and WT-1 on 23 of the 30 cases due to the difficulty in obtaining additional blank slides after the dropped tissue sections in the first 2 IHC attempts. As shown in Table 1, the CC and NBD of all cases (23/23, 100\% for both) were positive for CK19, a known pancreatobiliary marker. All CC and NBD were negative for WT-1 and D2-40, both known as mesothelial markers, except 1 NBD positive for WT-1 $(1 / 23,4.3 \%)$ and 1 CC positive for D2-40 (1/30, $3.3 \%)$. Those unexpected positive WT-1 and D2-40 stains were focal, and scored only $1+$ (less than $25 \%$, see Table 2 ). No significant difference of CK19, WT-1 or D2-40 IHC stain was found between the CC and NBD groups (Tables 1 and 2).

Of the $23 \mathrm{CC}$ cases evaluated for calretinin positivity, 12 (52.2\%, 95\% CI 30.6-73.2\%) were stained positive. CC tumor cells of the 12 cases showed dense and diffuse nuclear and cytoplasmic staining pattern of calretinin (Figure 1), while negative in the adjacent NBD in all cases $(P<0.001)$. For CK5/6 evaluation, $22(73 \%)$ of $\mathrm{CC}$ and all $30(100 \%)$ of NBD were positive (Table 2, Figure 2). Comparison of the CK5/6 expression in CC and the adjacent NBD revealed CC stained weaker than NBD in $18(60.0 \%)$ cases, similar to NBD in 10 (33.3\%), and stronger than NBD in 2 (6.67\%) (Tables 2 and 3). Eight out of the 30 CC cases (26.7\%, 95\% CI 12.3-45.9\%) were stained negative for CK5/6 while none of the NBD was negative $(P=0.002)$. The difference of CK5/6 in CC versus adjacent NBD is statistically significant $(P=0.022$, Table 3). Of note, the two cases with CC stained stronger than NBD had 3+ stains in CC and 2+ in NBD (25-50 staining area difference). In the meantime, 9 (50\%) of the 18 cases with CC stained weaker than NBD presented a difference of $2+$ or more (50-99\%) (Table 3).

We then sought the best IHC diagnostic criteria for differentiating $\mathrm{CC}$ versus $\mathrm{NBD}$ by comparing various 
Table 1 Immunohistochemical profiles of the cholangiocarcinoma and accompanying normal bile ducts

\begin{tabular}{|c|c|c|c|c|c|c|}
\hline & & $\begin{array}{l}\text { Negative, } \mathrm{n} \text { (mean } \% \text {, } \\
95 \% \text { confidence intervals) }\end{array}$ & $\begin{array}{l}\text { Positive, } \mathrm{n} \text { (mean } \% \text {, } \\
95 \% \text { confidence intervals) }\end{array}$ & Total, n (\%) & $P$ value* & $P$ value\# \\
\hline \multirow[t]{2}{*}{ Calretinin } & CC & $11(47.8,26.8-69.4)$ & $12(52.2,30.6-73.2)$ & $23(100)$ & $<0.001$ & NA \\
\hline & NBD & $23(100,85.2-100 \wedge)$ & $0(0)$ & $23(100)$ & & \\
\hline \multirow[t]{2}{*}{ WT1 } & $\mathrm{CC}$ & $23(100,85.2-100 \wedge)$ & $0(0)$ & $23(100)$ & 0.312 & 1 \\
\hline & NBD & $22(95.6,78.1-99.9)$ & $1(4.3,0.1-21.9)$ & $23(100)$ & & \\
\hline \multirow[t]{2}{*}{ D2-40 } & $\mathrm{CC}$ & $29(96.7,82.8-99.9)$ & $1(3.3,0-17.2)$ & $30(100)$ & 0.313 & 1 \\
\hline & NBD & $30(100,88.4-100 \wedge)$ & $0(0)$ & $30(100)$ & & \\
\hline \multirow[t]{2}{*}{ CK19 } & $\mathrm{CC}$ & $0(0)$ & $23(100,85.2-100 \wedge)$ & $23(100)$ & 0.312 & NA \\
\hline & NBD & $0(0)$ & $23(100,85.2-100 \wedge)$ & $23(100)$ & & \\
\hline \multirow[t]{2}{*}{ CK5/6 } & $\mathrm{CC}$ & $8(26.7,12.3-45.9)$ & $22(73.3,54.1-87.7)$ & $30(100)$ & 0.002 & NA \\
\hline & NBD & $0(0)$ & $30(100,88.4-100 \wedge)$ & $30(100)$ & & \\
\hline
\end{tabular}

Note: CC: Cholangiocarcinoma, NBD: accompanying normal bile duct, NA: not applicable, *comparison between CC and NBD by using Pearson Chi-square test, \#comparison between CC and NBD by by using Fischer's exact test, ^one-sided, $97.5 \%$ confidence interval.

cutoff values of the 2 markers (Table 4). The best sensitivity of $69.59 \%$ was achieved by using the sequential stains of calretin and CK5/6, with positive calretinin and negative CK5/6 in the calretinin negative cases as the positive result for $\mathrm{CC}$ (sequential calretinin and CK5/6 criteria), while maintaining $100 \%$ specificity. This sensitivity was statistically higher than that of calcretinin stain more than $2+$, or negative CK5/6 stain. However, we did not find the sensitivity difference between the sequential calretinin and CK5/6 criteria and the others, including positive calretinin stain alone (52.17\%), CK5/6 stained negative or $1+(60.0 \%)$ and less CK5/6 stain in CC than NBD (56.67\%) (Table 4). Our Fisher exact test also revealed that CK5/6 expression was more likely associated with well-differentiated $\mathrm{CC}$ and extrahepatic $\mathrm{CC}$, but no association between the calretinin expression and the $\mathrm{CC}$ grade or location (Table 5 ).

\section{Discussion}

Cholangiocarcinoma is an uncommon carcinoma in the developed countries, but had a rising mortality in both UK and USA [1,2]. Its carcinogenesis and diagnostic markers are not well defined. Studies have revealed some IHC markers such as CK7, CK19, MOC31, claudin4, HMGA, CD133 and Annexin A1, but with variable specificities (Table 6) [3-5,13]. In particular, little is known about the IHC marks' expression in NBD. To our best knowledge, this study is the first to explore the use of a set of known mesothelial markers for differentiating CC from accompanying NBD.

Calretinin is a $29-30$ kilodalton calcium binding protein primarily expressed in the nerves [33]. Since it was found expressed in mesothelioma in 1990s [34], many types of tumor and tissue are found immunocreactive to calretinin including $22.5 \%$ of examined colonic carcinomas [35], $81.5 \%$ of ameloblastomas [36], 36\% of thymic carcinomas [10], 100\% of cardiac myxomas [37], 56-100\% sex cord-stromal and $90-100 \%$ fibrous neoplasms of the ovaries [38-40], 95\% olfactory neuroblastoma [41], 95\% of adrenal cortical tumors [42], 71\% of synovial sarcomas [43], 15\% breast carcinomas [44], skin [45] and others [46]. Our study indicates that CC may be calretinin positive regardless of $\mathrm{CC}$ grade and location, and should be considered in the differential diagnoses for calretinin positive tumor. As many as 52\% of our CC cases showed strong nuclear and cytoplasmic calretinin expression, while none in the NBD, suggesting a potential role of calretinin in differentiating CC from NBD and in CC carcinogenesis (Table 4). In contrast, CK19 positivity does not discriminate $\mathrm{CC}$ and non-cancerous NBD, and should

Table 2 Immunohistochemical stain scores of the cholangiocarcinoma and accompanying normal bile ducts

\begin{tabular}{|c|c|c|c|c|c|c|c|c|c|c|}
\hline & \multicolumn{2}{|c|}{ Calretinin, n (\%) } & \multicolumn{2}{|c|}{ WT1, n (\%) } & \multicolumn{2}{|c|}{ D2-40, n (\%) } & \multicolumn{2}{|c|}{ CK19, n (\%) } & \multicolumn{2}{|c|}{ CK5/6, n (\%) } \\
\hline & $\overline{C C}$ & NBD & $\overline{C C}$ & NBD & $\overline{C C}$ & NBD & $\overline{C C}$ & NBD & $\overline{C C}$ & NBD \\
\hline Negative & $11(47.8)$ & $23(100)$ & $23(100)$ & $22(95.6)$ & $29(96.7)$ & $30(100)$ & $0(0)$ & $0(0)$ & $8(26.7)$ & $0(0)$ \\
\hline $1+$ & $4(30.8)$ & $0(0)$ & $0(0)$ & $1(4.3)$ & $1(3.3)$ & $0(0)$ & $0(0)$ & $0(0)$ & $10(33.3)$ & $8(26.7)$ \\
\hline $2+$ & $4(30.8)$ & $0(0)$ & $0(0)$ & $0(0)$ & $0(0)$ & $0(0)$ & $1(4.3)$ & $0(0)$ & $4(13.3)$ & $7(23.3)$ \\
\hline $3+$ & $4(30.8)$ & $0(0)$ & $0(0)$ & $0(0)$ & $0(0)$ & $0(0)$ & $22(95.6)$ & $23(100)$ & $8(26.7)$ & $15(50)$ \\
\hline All Positive & $12(52.2)$ & $0(0)$ & $0(0)$ & $1(4.3)$ & $1(3.3)$ & $0(0)$ & $23(100)$ & $23(100)$ & $22(73.3)$ & $30(100)$ \\
\hline total & $23(100)$ & $23(100)$ & $23(100)$ & $23(100)$ & $30(100)$ & $30(100)$ & $23(100)$ & $23(100)$ & $30(100)$ & $30(100)$ \\
\hline
\end{tabular}

Note: CC: Cholangiocarcinoma, NBD: accompanying normal bile duct. 


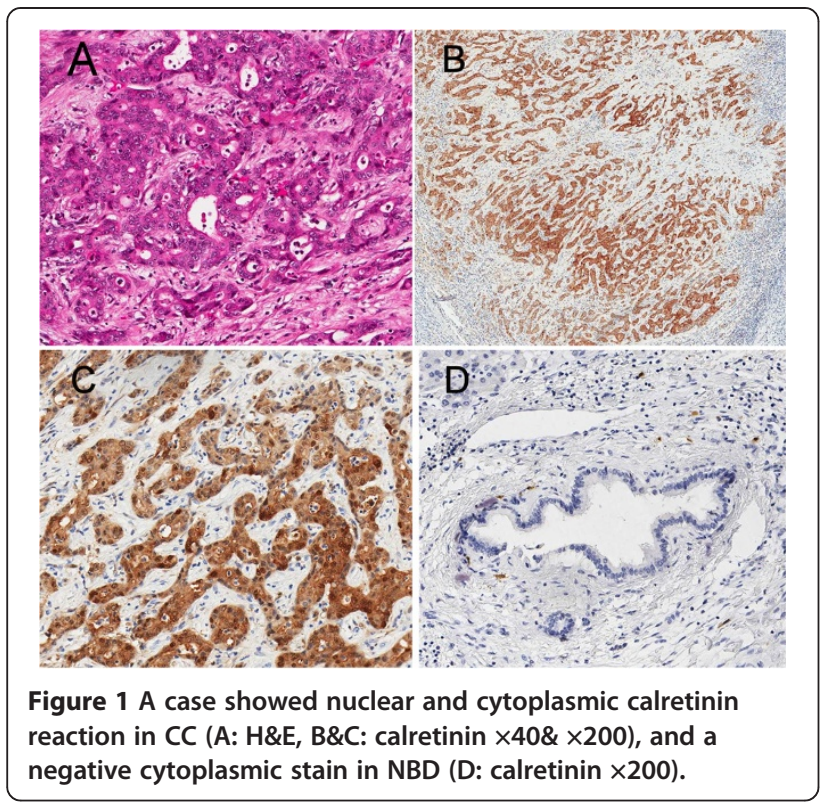

be used only for confirmation of a bile duct (biliary) lineage.

We also examined the expression of other mesothelial markers in CC and NBD including WT1, D2-40 and CK5/6 [12,30,47-49]. Not surprisingly, our data showed that WT1 and D2-40 remained highly specific for mesothelial lineage, and should be included in the panel to differentiate mesothelioma from CC. Of note, WT1 has also been reported positive in more than $90 \%$ in adenomatoid tumors [50], 76\% ovarian sex cord-stromal tumor [39], 77\% serous papillary carcinoma of the ovary [40], and $29 \%$ of endometrioid carcinoma [40]. Caution

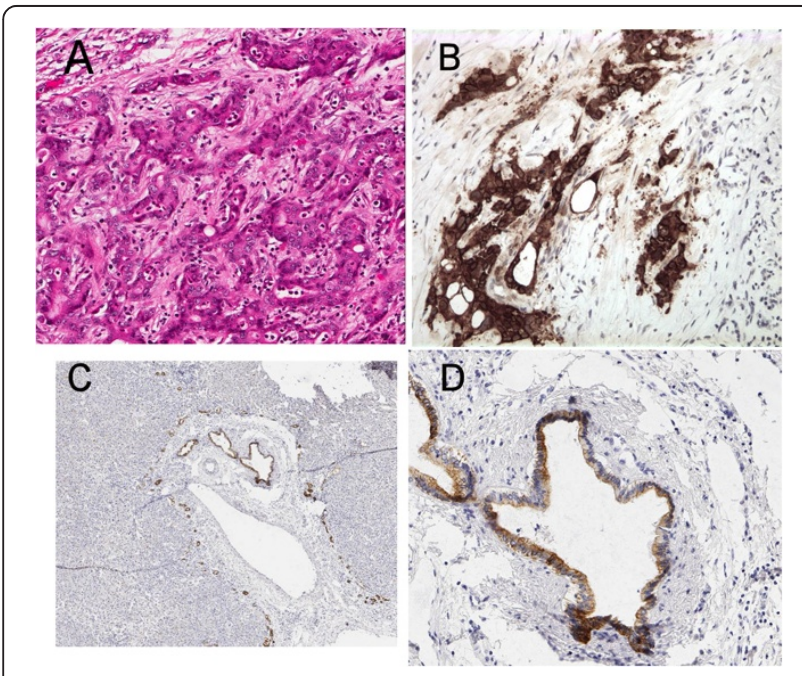

Figure $2 \mathrm{~A}$ case showed nuclear and cytoplasmic CK5/6 reaction in CC (A: H\&E, B: CK5/6 $\times 200)$ and a weaker cytoplasmic stain in a NBD (C\&D: CK5/6 ×40\& $\times 200)$.
Table 3 Comparison of CK5/6 stains between cholangiocarinoma and normal bile duct

\begin{tabular}{|c|c|c|c|c|}
\hline & $\mathrm{CC}$ & & & \\
\hline NBD & 0 & 1 & 2 & 3 \\
\hline \multicolumn{5}{|l|}{0} \\
\hline 1 & 3 & 4 & & \\
\hline 2 & 3 & 2 & & 2 \\
\hline 3 & 2 & 4 & 4 & 6 \\
\hline
\end{tabular}

Note: Stain intensity comparison: CC < NBD: 18, CC $=$ NBD: 10, and CC > NBD: 2 $(P=0.022)$; CC: Cholangiocarcinoma, NBD: accompanying normal bile duct. The number in bold shows the case number with stain difference of more than $1+$ ( $>25 \%$ difference).

therefore should be used when interpreting a WT1 positive stain, particularly in female patients. Addition of D2-40 may also improve the sensitivity and specificity for confirming mesothelial differentiation [12].

CK5/6 is a high molecular-weight cytokeratin highly expressed in stratified epithelium and mesothelium, first found useful for distinguishing mesothelioma from adenocarcinoma in late 1990s [51,52]. Its positive rate in mesothelioma is comparable to that of calretinin [20]. A recent systemic review also confirms that CK5/6 is one of the two most sensitive IHC markers (sensitivity of $83 \%$ ), and one of the two most specific IHC markers (specificity of $85 \%$ ) for epitheloid mesothelioma [30]. However, many tumors other than mesothelioma are also positive for CK5/6, including but not limited to $88 \%$ of adenosquamous carcinoma of the pancreas [53], 55\% of metastatic squamous carcinoma of various origin [54], $75 \%$ of lung squamous cell carcinoma in fine needle aspirate specimens [55], $100 \%$ of squamous cell carcinoma in pleural fluids [14], $98 \%$ of squamous cell carcinoma and $18 \%$ of adenocarcinoma in the lung [56], 62\% of urothelial carcinoma [57], and 50\% of endometrial adenocarcinoma [57]. Our study showed that all NBD and $73.3 \%$ of CC were positive for $\mathrm{CK} 5 / 6$. Further analysis found that CK5/6 expression was significant lower in the CC than the accompanying NBD (Table 3). Those findings demonstrate the differential expressions of CK5/6 in CC and NBD, and suggest a potential use of CK5/6 in differentiating CC from NBD. The association between CK5/6 expression and extrahepatic and well-differentiated $\mathrm{CC}$ indicates a preference of using CK5/6 in those CCs.

Of the markers we tested, the best sensitivity to identify $\mathrm{CC}$ is reached by using sequential criteria of positive calretinin and negative CK5/6 in the calretinin negative cases, with a $100 \%$ specificity. Using positive calretinin stain criterion is straight forward, and will result in only a $17.47 \%$ loss of sensitivity, or missing 4 out of 23 cases as shown in our study. This may be the second best strategy of using calretinin and/or CK5/6 to differentiate CC from NBD. The criterion of less CK5/6 stain in tumor than NBD requires presence of both tumor and NBD in 
Table 4 Comparison of various calretinin and CK5/6 diagnostic criteria for cholangiocarcinoma

\begin{tabular}{|c|c|c|c|c|c|c|c|c|c|}
\hline No. & Criteria & CC & Total CC & Sensitivity & $P$ value* & NBD & Total NBD & Specificity & $P$ value* \\
\hline 1 & Cal 1+ & 12 & 23 & $52.17 \%$ & 0.227 & 23 & 23 & $100.00 \%$ & NA \\
\hline 2 & Cal 2+ & 8 & 23 & $34.78 \%$ & 0.018 & 23 & 23 & $100.00 \%$ & NA \\
\hline 3 & Cal 3+ & 4 & 23 & $17.39 \%$ & $<0.001 \#$ & 23 & 23 & $100.00 \%$ & NA \\
\hline 4 & CK5/6 - & 8 & 30 & $26.67 \%$ & 0.002 & 30 & 30 & $100.00 \%$ & NA \\
\hline 5 & CK5/6 - or $1+$ & 18 & 30 & $60.00 \%$ & 0.472 & 22 & 30 & $73.33 \%$ & 0.007 \\
\hline 6 & Less $C K 5 / 6$ staining in $C C$ than in NBD & 17 & 30 & $56.67 \%$ & 0.337 & & & NA & \\
\hline 7 & Cal+, and CK5/6 - in Cal - cases & 16 & 23 & $69.57 \%$ & & 23 & 23 & $100.00 \%$ & \\
\hline
\end{tabular}

Note: Cal: calretinin, CC: Cholangiocarcinoma, NBD: accompanying normal bile duct, NA: not applicable, *Comparison between criteria 7 and other criteria by using Pearson Chi-square test, \#Comparison between criteria 7 and 5 by using Fischer's exact test.

the same specimens, which would be particularly challenging in core biopsies and cytology specimens. We therefore doubt its clinical usefulness due to specimen type limitations. Negative or $1+$ CK5/6 stain gave us the second highest sensitivity, but also led to a significantly lower specificity $(100 \%$ vs $73.3 \%, P=0.007)$. Despite its single IHC stain requirement, we felt the lower specificity would be a serious concern for its clinical use. Together, we recommend sequential staining pattern of positive calretinin and negative CK5/6 in the calretinin negative cases for differentiating CC from NBD. Future work may focus on larger-scale studies and differentiating $\mathrm{CC}$ from $\mathrm{HCC}$ and mesothelioma by using these criteria.

We summarized the expression profiles of the common IHC markers for CC, mesothelioma and HCC in Table 6. Interestingly, the positive rates of those markers vary among studies. For example, the positive rate of MOC31 in mesothelioma ranged from $8 \%$ to $35 \%$. Consistent with our summary, an excellent review and another guidelines have confirmed such a variation among reports, and made the practical yet useful recommendations on how to best utilize those markers $[15,48,58]$. We suggest to include at least WT1 and D2-40 in the panel to confirm a mesothelial lineage, with optional addition of calretinin, CK5/6 and/or mesothelin. Should WT1 and/or D2-40 stain be not interpretable due to technical issues or limitations (such as a fallen section), one must use other mesothelial markers to rule out the tumors with calretinin and CK5/6 reactivity from mesothelioma, such as CC as shown in this study. The reported different positive rates of the IHC markers may be in part attributed to the various IHC staining methods. For example, we noticed that CK5/6 expression was present in $2 \%$ of lung adenocarcinoma by using 1:25 dilution of D5/16B4 antibody (Boehringer-Mannheim) and Envision + biotin free detection system in Dako AutoStainer [16], but 39\% by using the same 1:25 dilution of different D5/16B4 antibody (Dako) and Envision + HRP detection system in the same IHC stainer [12]. Similarly, using different dilutions of calretinin antibody (rabbit,

Table 5 Association of the cholangiocarcinoma grades and locations with calrectinin and CK5/6 expression

\begin{tabular}{|c|c|c|c|c|c|c|c|c|c|c|c|c|c|c|c|c|c|c|}
\hline & \multirow[b]{2}{*}{ Neg } & \multicolumn{2}{|c|}{ Calretintin } & \multirow[b]{2}{*}{$3+$} & \multirow[b]{2}{*}{$P$} & \multicolumn{3}{|c|}{ Calretinin } & \multirow[b]{2}{*}{$P$} & \multirow[b]{2}{*}{ Neg } & \multirow[b]{2}{*}{$1+$} & \multicolumn{2}{|c|}{ CK 5/6 } & \multirow[b]{2}{*}{$P$} & \multicolumn{3}{|c|}{ CK5/6 } & \multirow[b]{2}{*}{$P$} \\
\hline & & $1+$ & $2+$ & & & $\mathrm{Neg}$ & Pos & Sum & & & & $2+$ & $3+$ & & Neg & Pos & Sum & \\
\hline WD & 3 & 1 & 2 & 1 & $\#$ & 3 & 4 & 7 & $\#$ & 0 & 2 & 3 & 2 & 0.032 & 0 & 7 & 7 & 0.019 \\
\hline$\%$ & 42.86 & 14.29 & 28.57 & 14.29 & & 43 & 57.1 & 100 & & 0 & 28.57 & 42.86 & 28.57 & & 0 & 100 & 100 & \\
\hline $\mathrm{MD}$ & 6 & 2 & 2 & 0 & & 6 & 4 & 10 & & 7 & 4 & 0 & 2 & & 7 & 6 & 13 & \\
\hline$\%$ & 60 & 20 & 20 & 0 & & 60 & 40 & 100 & & 53.85 & 30.77 & 0 & 15.38 & & 53.85 & 46.15 & 100 & \\
\hline PD & 2 & 1 & 0 & 3 & & 2 & 4 & 6 & & 1 & 4 & 1 & 4 & & 1 & 9 & 10 & \\
\hline$\%$ & 33.33 & 16.67 & 0 & 50 & & 33 & 66.7 & 100 & & 10 & 40 & 10 & 40 & & 10 & 90 & 100 & \\
\hline $\mathrm{EH}$ & 3 & 0 & 3 & 1 & $\#$ & 3 & 4 & 7 & $\#$ & 0 & 4 & 0 & 6 & 0.004 & 0 & 10 & 10 & 0.029 \\
\hline$\%$ & 42.86 & 0 & 42.86 & 14.29 & & 43 & 57.1 & 100 & & 0 & 40 & 0 & 60 & & 0 & 100 & 100 & \\
\hline$\| \mathrm{H}$ & 8 & 4 & 1 & 3 & & 8 & 8 & 16 & & 8 & 6 & 4 & 2 & & 8 & 12 & 20 & \\
\hline$\%$ & 50 & 25 & 6.25 & 18.75 & & 50 & 50 & 100 & & 40 & 30 & 20 & 10 & & 40 & 60 & 100 & \\
\hline Sum & 11 & 4 & 4 & 4 & & 11 & 12 & 23 & & 8 & 10 & 4 & 8 & & 8 & 22 & 30 & \\
\hline$\%$ & 47.83 & 17.39 & 17.39 & 17.39 & & 48 & 52.2 & 100 & & 26.67 & 33.33 & 13.33 & 26.67 & & 26.67 & 73.33 & 100 & \\
\hline
\end{tabular}

Notes: $\mathrm{WD}=$ well-differentiated, $\mathrm{MD}=$ moderately-differentiated, $\mathrm{PD}=$ Poorly-differentiated, $\mathrm{EH}=$ extrahepatic cholangiocarcinoma, $\mathrm{IH}=$ intrahepatic cholangiocarcinoma, $P$ : $P$-value, \#: $P>0.005$. 
Table 6 Positive rates of immunohistochemical markers for mesothelioma, cholangiocarcinoma, lung adenocarcinoma and hepatocellular carcinoma

\begin{tabular}{|c|c|c|c|c|}
\hline & Mesothelioma (all subtypes included) & Cholangiocarcinoma & Lung adenocarcinoma & Hepatocellular carcinoma \\
\hline WT-1 & $43-93 \%[11,12,14-17]$ & $0 \% \#$ & $0-7 \%[12,14,16]$ & NA \\
\hline D2-40 & $86-100 \%[12,18,19]$ & $3 \% \#$ & $0-33 \%[12,18,19]$ & NA \\
\hline Calretinin & $92.4-100 \%[11,12,16,17,20,21]$ & $52 \% \#$ & $8-23 \%[12,16,22]$ & NA \\
\hline CK5/6 & $64-100 \%[11,12,15-17,20]$ & 0-73.3\%\#, [23] & $0-39 \%[12,14-16,23]$ & NA \\
\hline Mesothelin & $47-100 \%[14,16,17]$ & $33 \%[7]$ & $38-100 \%[14,16,22]$ & NA \\
\hline CK19 & NA & 89-100\#, [3,24] & NA & $2-10.1 \%[3,24]$ \\
\hline Annexin A1 & NA & $94.1 \%[5]$ & NA & $0 \%[5]$ \\
\hline Glypican-3 & NA & $6-7 \%[3,24]$ & $3.6-9.6 \%[25,26]$ & $69-87.1 \%[3,24-26]$ \\
\hline Arginase & NA & $0 \%[24]$ & $0 \%[26]$ & $94-95 \%[24,26]$ \\
\hline TTF-1 & $0 \%[16]$ & $0-10 \%[23,27]$ & (nuclear) $20-74 \%[16,23]$ & (cytoplasmic) 50-93\% [27-29] \\
\hline HepPar-1 & NA & $0-7 \%[23,24]$ & $8.1 \%[26]$ & $74-100 \%[23,24,26]$ \\
\hline MOC31 & $8-35 \%[14,16]$ & $88.2 \%$ [3] & $92-100 \%[14,16,17,30]$ & $34.0 \%[3]$ \\
\hline EMA & $79-93 \%[16,21]$ & $100 \%[31,32]$ & $100 \%[16]$ & $12.5-23 \%[31,32]$ \\
\hline BG 8 & $7 \%[16]$ & NA & $95-96 \%[16,17]$ & NA \\
\hline BerEP4 & $16-18 \%[16,21]$ & $100 \%[23]$ & $74-100 \%[16,17]$ & $33 \%$ [23] \\
\hline
\end{tabular}

Note: \#indicates this study; Sensitivity reported in some studies [17] is included; NA: No IHC data on the respective marker are identified by searching the Pubmed database.

Zymed, South San Francisco, CA) seemed to result in different calretinin positive rates in lung adenocarcinoma $(8 \%$ versus $23 \%)[11,12,16]$. Another study also showed that the TTF-1 positive rates in hepatocellular carcinomas vary from $0 \%$ to $70 \%$ depending on the antibody manufacturer [28]. An in-house validation of new antibodies on various tumors seems a reasonable safe-guard approach and is recommended.

On the cancer biology level, calretinin has higher positive rates in CC than mesothelin (52.17\% versus 33\%), and may also be a more sensitive and/or specific therapeutic target for CC. However, little is known regarding the roles of calrectin and CK5/6 in the carcinogenesis of $\mathrm{CC}$ and the biology of biliary epithelium. Given the recent identification of both mesothelial progenitor cells and liver stem cells [59-62], we hypothesize that the expression of calretinin and CK5/6 in CC is an aberrant differentiation of liver/bile duct stem cells, or simply reflecting the partial mesothelial phenotype of the NBD. However, much research is needed to examine our hypothesis.

This study has several limitations. First, this retrospective study may have selection bias and moderate statistical power. Second, the calretinin and CK5/6 staining profiles were not compared between $\mathrm{CC}$, mesothelioma and HCC. A direct comparison of calretinin and CK5/6 expression in those tumors would be more evidential. Third, the calretinin and CK5/6 IHC patterns in ductal proliferation including reactive changes or benign ductal neoplasms were not assessed, but may be of particular value in differentiating carcinoma and non-cancerous lesions. Last, the prognostic value of calretinin and CK5/ 6 expression in CC is not explored in this study, but may be interesting to investigate because another mesothelial marker, mesothelin, has been considered for therapeutic targets for CC. Survival studies are beyond the scope of this study, however. Future work is needed to address the unanswered questions and our study's limitations.

\section{Conclusions}

In conclusion, we for first time showed calretinin and CK5/6 expression in CC. The sequential criterion of positive calretinin stain and negative CK5/6 stain in calretinin negative cases has a sensitivity of $69.57 \%$ and a specificity of $100 \%$ for differentiating CC from NBD. Our data also suggest to include at least one or two markers more specific for mesothelial differentiation, such as D2-40 and WT1, in the panel to define a mesothelial lineage.

\section{Abbreviations \\ CC: Cholangiocarcinoma; IHC: Immunohistochemical; GPC3: Glypican 3; NBD: Normal bile duct; Cl: Confidence intervals.}

\section{Competing interest}

The authors declared that they have no competing interests.

\section{Authors' contributions}

LZ and PJZ, designed the study, conducted analyses, interpreted results and drafted the manuscript. LZ, AFZ and RF conducted the study and analyses. EFF and VAL reviewed the study design, interpreted results and were involved in manuscript development. All authors read and approved the final manuscript. 


\section{Acknowledgments}

We thank Kerry A. O'Rourke at Rutgers Robert Wood Johnson Library of Health Sciences for her assistance in literature retrieval. Despite of our efforts to include all publications related to the topics, we may have inadvertently omitted some and would like to apologize to their authors.

\section{Author details}

${ }^{1}$ Department of Pathology and Laboratory Medicine, University of Pennsylvania Pearlman School of Medicine, Philadelphia, PA, USA ${ }^{2}$ Departments of Pathology, University Medical Center of Princeton at Plainsboro/Rutgers Robert Wood Johnson Medical School, Plainsboro, NJ, USA. ${ }^{3}$ Department of Chemical Biology, Ernest Mario School of Pharmacy, Department of Pathology and Lab Medicine, Robert Wood Johnson Medica School, and Cancer Institute of New Jersey, Rutgers University, Piscataway, NJ, USA. ${ }^{4}$ Department of Pathology, 6 Founders, 3400 Spruce St, Philadelphia, PA 19104, USA.

Received: 24 February 2014 Accepted: 9 April 2014

Published: 23 April 2014

\section{References}

1. Patel T: Increasing incidence and mortality of primary intrahepatic cholangiocarcinoma in the United States. Hepatology 2001, 33:1353-1357

2. Taylor-Robinson SD, Toledano MB, Arora S, Keegan TJ, Hargreaves S, Beck A, Khan SA, Elliott $P$, Thomas HC: Increase in mortality rates from intrahepatic cholangiocarcinoma in England and Wales 1968-1998. Gut 2001, 48:816-820.

3. Ryu HS, Lee K, Shin E, Kim SH, Jing J, Jung HY, Lee H, Jang JJ: Comparative analysis of immunohistochemical markers for differential diagnosis of hepatocelluar carcinoma and cholangiocarcinoma. Tumori 2012, 98:478-484

4. Fan L, He F, Liu H, Zhu J, Liu Y, Yin Z, Wang L, Guo Y, Wang Z, Yan Q, Huang G: CD133: a potential indicator for differentiation and prognosis of human cholangiocarcinoma. BMC Cancer 2011, 11:320.

5. Hongsrichan N, Rucksaken R, Chamgramol Y, Pinlaor P, Techasen A, Yongvanit P, Khuntikeo N, Pairojkul C, Pinlaor S: Annexin A1: A new immunohistological marker of cholangiocarcinoma. World J Gastroenterol 2013, 19:2456-2465

6. Sato Y, Harada K, Sasaki M, Nakanuma Y: Clinicopathological significance of S100 protein expression in cholangiocarcinoma. J Gastroenterol Hepatol 2013, 28:1422-1429.

7. Yu L, Feng M, Kim H, Phung Y, Kleiner DE, Gores GJ, Qian M, Wang XW, Ho M: Mesothelin as a potential therapeutic target in human cholangiocarcinoma. J Cancer 2010, 1:141-149.

8. Ho M: Advances in liver cancer antibody therapies: a focus on glypican-3 and mesothelin. BioDrugs 2011, 25:275-284.

9. Kawamata F, Kamachi H, Einama T, Homma S, Tahara M, Miyazaki M, Tanaka S, Kamiyama T, Nishihara H, Taketomi A, Todo S: Intracellular localization of mesothelin predicts patient prognosis of extrahepatic bile duct cancer. Int J Oncol 2012, 41:2109-2118.

10. Pan CC, Chen PC, Chou TY, Chiang H: Expression of calretinin and other mesothelioma-related markers in thymic carcinoma and thymoma. Hum Pathol 2003, 34:1155-1162.

11. Ordonez NG: The diagnostic utility of immunohistochemistry in distinguishing between epithelioid mesotheliomas and squamous carcinomas of the lung: a comparative study. Mod Pathol 2006, 19:417-428.

12. Chu AY, Litzky LA, Pasha TL, Acs G, Zhang PJ: Utility of D2-40, a nove mesothelial marker, in the diagnosis of malignant mesothelioma. Mod Pathol 2005, 18:105-110.

13. Jain R, Fischer S, Serra S, Chetty R: The use of Cytokeratin 19 (CK19) immunohistochemistry in lesions of the pancreas, gastrointestinal tract, and liver. Appl Immunohistochem Mol Morphol 2010, 18:9-15.

14. Pu RT, Pang Y, Michael CW: Utility of WT-1, p63, MOC31, mesothelin, and cytokeratin (K903 and $\mathrm{CK} 5 / 6$ ) immunostains in differentiating adenocarcinoma, squamous cell carcinoma, and malignant mesothelioma in effusions. Diagn Cytopathol 2008, 36:20-25.

15. Ordonez NG: Immunohistochemical diagnosis of epithelioid mesothelioma: an update. Arch Pathol Lab Med 2005, 129:1407-1414.
16. Ordonez NG: The immunohistochemical diagnosis of mesothelioma: a comparative study of epithelioid mesothelioma and lung adenocarcinoma. Am J Surg Pathol 2003, 27:1031-1051.

17. Yaziji H, Battifora H, Barry TS, Hwang HC, Bacchi CE, Mclntosh MW, Kussick SJ, Gown AM: Evaluation of 12 antibodies for distinguishing epithelioid mesothelioma from adenocarcinoma: identification of a three-antibody immunohistochemical panel with maximal sensitivity and specificity. Mod Pathol 2006, 19:514-523.

18. Ordonez NG: D2-40 and podoplanin are highly specific and sensitive immunohistochemical markers of epithelioid malignant mesothelioma. Hum Pathol 2005, 36:372-380.

19. Bassarova AV, Nesland JM, Davidson B: D2-40 is not a specific marker for cells of mesothelial origin in serous effusions. Am J Surg Pathol 2006 30:878-882.

20. Cury PM, Butcher DN, Fisher C, Corrin B, Nicholson AG: Value of the mesothelium-associated antibodies thrombomodulin, cytokeratin 5/6, calretinin, and $\mathrm{CD} 44 \mathrm{H}$ in distinguishing epithelioid pleural mesothelioma from adenocarcinoma metastatic to the pleura. Mod Pathol 2000, 13:107-112.

21. Brockstedt U, Gulyas M, Dobra K, Dejmek A, Hjerpe A: An optimized battery of eight antibodies that can distinguish most cases of epithelial mesothelioma from adenocarcinoma. Am J Clin Pathol 2000, 114:203-209.

22. Miettinen $M$, Sarlomo-Rikala M: Expression of calretinin, thrombomodulin keratin 5, and mesothelin in lung carcinomas of different types: an immunohistochemical analysis of 596 tumors in comparison with epithelioid mesotheliomas of the pleura. Am J Surg Pathol 2003, 27:150-158.

23. Onofre AS, Pomjanski N, Buckstegge B, Bocking A: Immunocytochemical diagnosis of hepatocellular carcinoma and identification of carcinomas of unknown primary metastatic to the liver on fine-needle aspiration cytologies. Cancer 2007, 111:259-268

24. Krings G, Ramachandran R, Jain D, Wu T, Yeh MM, Torbenson M, Kakar S: Immunohistochemical pitfalls and the importance of glypican 3 and arginase in the diagnosis of scirrhous hepatocellular carcinoma. Mod Pathol 2013, 26:782-791.

25. Zhang L, Liu H, Sun L, Li N, Ding H, Zheng J: Glypican-3 as a potential differential diagnosis marker for hepatocellular carcinoma: a tissue microarray-based study. Acta Histochem 2012, 114:547-552.

26. Timek DT, Shi J, Liu H, Lin F: Arginase-1, HepPar-1, and Glypican-3 are the most effective panel of markers in distinguishing hepatocellular carcinoma from metastatic tumor on fine-needle aspiration specimens. Am J Clin Pathol 2012, 138:203-210.

27. Lei JY, Bourne PA, diSant' Agnese PA, Huang J: Cytoplasmic staining of TTF-1 in the differential diagnosis of hepatocellular carcinoma vs cholangiocarcinoma and metastatic carcinoma of the liver. Am J Clin Pathol 2006, 125:519-525.

28. Pan CC, Chen PC, Tsay SH, Chiang H: Cytoplasmic immunoreactivity for thyroid transcription factor-1 in hepatocellular carcinoma: a comparative immunohistochemical analysis of four commercial antibodies using a tissue array technique. Am J Clin Pathol 2004, 121:343-349.

29. Wieczorek TJ, Pinkus JL, Glickman JN, Pinkus GS: Comparison of thyroid transcription factor-1 and hepatocyte antigen immunohistochemical analysis in the differential diagnosis of hepatocellular carcinoma, metastatic adenocarcinoma, renal cell carcinoma, and adrenal cortical carcinoma. Am J Clin Pathol 2002, 118:911-921.

30. King JE, Thatcher N, Pickering CA, Hasleton PS: Sensitivity and specificity of immunohistochemical markers used in the diagnosis of epithelioid mesothelioma: a detailed systematic analysis using published data. Histopathology 2006, 48:223-232.

31. Bonetti F, Chilosi M, Pisa R, Novelli P, Zamboni G, Menestrina F: Epithelial membrane antigen expression in cholangiocarcinoma. An useful immunohistochemical tool for differential diagnosis with hepatocarcinoma. Virchows Arch A Pathol Anat Histopathol 1983, 401:307-313.

32. Tsuji M, Kashihara T, Terada N, Mori H: An immunohistochemical study of hepatic atypical adenomatous hyperplasia, hepatocellular carcinoma, and cholangiocarcinoma with alpha-fetoprotein, carcinoembryonic antigen, CA19-9, epithelial membrane antigen, and cytokeratins 18 and 19. Pathol Int 1999, 49:310-317.

33. Rogers J, Khan M, Ellis J: Calretinin and other CaBPs in the nervous system. Adv Exp Med Biol 1990, 269:195-203. 
34. Doglioni C, Dei Tos AP, Laurino L, luzzolino P, Chiarelli C, Celio MR, Viale G: Calretinin: a novel immunocytochemical marker for mesothelioma. Am J Surg Pathol 1996, 20:1037-1046.

35. Gotzos V, Wintergerst ES, Musy JP, Spichtin HP, Genton CY: Selective distribution of calretinin in adenocarcinomas of the human colon and adjacent tissues. Am J Surg Pathol 1999, 23:701-711.

36. Altini M, Coleman H, Doglioni C, Favia G, Maiorano E: Calretinin expression in ameloblastomas. Histopathology 2000, 37:27-32

37. Terracciano LM, Mhawech P, Suess K, D'Armiento M, Lehmann FS, Jundt G, Moch H, Sauter G, Mihatsch MJ: Calretinin as a marker for cardiac myxoma. Diagnostic and histogenetic considerations. Am J Clin Pathol 2000, 114:754-759.

38. Movahedi-Lankarani S, Kurman RJ: Calretinin, a more sensitive but less specific marker than alpha-inhibin for ovarian sex cord-stromal neoplasms: an immunohistochemical study of 215 cases. Am J Surg Pathol 2002, 26:1477-1483.

39. Deavers MT, Malpica A, Liu J, Broaddus R, Silva EG: Ovarian sex cordstromal tumors: an immunohistochemical study including a comparison of calretinin and inhibin. Mod Pathol 2003, 16:584-590.

40. Cathro HP, Stoler MH: The utility of calretinin, inhibin, and WT1 immunohistochemical staining in the differential diagnosis of ovarian tumors. Hum Pathol 2005, 36:195-201.

41. Wooff JC, Weinreb I, Perez-Ordonez B, Magee JF, Bullock MJ: Calretinin staining facilitates differentiation of olfactory neuroblastoma from other small round blue cell tumors in the sinonasal tract. Am J Surg Pathol 2011, 35:1786-1793.

42. Zhang PJ, Genega EM, Tomaszewski JE, Pasha TL, LiVolsi VA: The role of calretinin, inhibin, melan-A, BCL-2, and C-kit in differentiating adrenal cortical and medullary tumors: an immunohistochemical study. Mod Pathol 2003, 16:591-597.

43. Miettinen M, Limon J, Niezabitowski A, Lasota J: Calretinin and other mesothelioma markers in synovial sarcoma: analysis of antigenic similarities and differences with malignant mesothelioma. Am J Surg Pathol 2001, 25:610-617.

44. Powell G, Roche $H$, Roche WR: Expression of calretinin by breast carcinoma and the potential for misdiagnosis of mesothelioma. Histopathology 2011, 59:950-956.

45. Gonzalez-Guerra E, Kutzner H, Rutten A, Requena L: Immunohistochemical study of calretinin in normal skin and cutaneous adnexal proliferations. Am J Dermatopathol 2012, 34:491-505.

46. Lugli A, Forster $Y$, Haas P, Nocito A, Bucher C, Bissig H, Mirlacher M, Storz M, Mihatsch MJ, Sauter G: Calretinin expression in human normal and neoplastic tissues: a tissue microarray analysis on 5233 tissue samples. Hum Pathol 2003, 34:994-1000.

47. Hyun TS, Barnes M, Tabatabai ZL: The diagnostic utility of D2-40, calretinin, CK5/6, desmin and MOC-31 in the differentiation of mesothelioma from adenocarcinoma in pleural effusion cytology. Acta Cytol 2012, 56:527-532.

48. Mohammad T, Garratt J, Torlakovic E, Gilks B, Churg A: Utility of a CEA, CD15, calretinin, and CK5/6 panel for distinguishing between mesotheliomas and pulmonary adenocarcinomas in clinical practice. Am J Surg Pathol 2012, 36:1503-1508.

49. Kaufmann O, Fietze E, Mengs J, Dietel M: Value of p63 and cytokeratin 5/6 as immunohistochemical markers for the differential diagnosis of poorly differentiated and undifferentiated carcinomas. Am J Clin Pathol 2001 116:823-830.

50. Sangoi AR, McKenney JK, Schwartz EJ, Rouse RV, Longacre TA: Adenomatoid tumors of the female and male genital tracts: a clinicopathological and immunohistochemical study of 44 cases. Mod Pathol 2009, 22:1228-1235

51. Ordonez NG: Value of cytokeratin $5 / 6$ immunostaining in distinguishing epithelial mesothelioma of the pleura from lung adenocarcinoma. Am Surg Pathol 1998, 22:1215-1221.

52. Clover J, Oates J, Edwards C: Anti-cytokeratin 5/6: a positive marker for epithelioid mesothelioma. Histopathology 1997, 31:140-143.

53. Kardon DE, Thompson LD, Przygodzki RM, Heffess CS: Adenosquamous carcinoma of the pancreas: a clinicopathologic series of 25 cases. Mod Pathol 2001, 14:443-451

54. Suo Z, Holm R, Nesland JM: Squamous cell carcinomas. An immunohistochemical study of cytokeratins and involucrin in primary and metastatic tumours. Histopathology 1993, 23:45-54.
55. Khayyata S, Yun S, Pasha T, Jian B, McGrath C, Yu G, Gupta P, Baloch Z: Value of P63 and CK5/6 in distinguishing squamous cell carcinoma from adenocarcinoma in lung fine-needle aspiration specimens. Diagn Cytopathol 2009, 37:178-183.

56. Rekhtman N, Ang DC, Sima CS, Travis WD, Moreira AL: Immunohistochemical algorithm for differentiation of lung adenocarcinoma and squamous cell carcinoma based on large series of whole-tissue sections with validation in small specimens. Mod Pathol 2011, 24:1348-1359.

57. Chu PG, Weiss LM: Expression of cytokeratin 5/6 in epithelial neoplasms: an immunohistochemical study of 509 cases. Mod Pathol 2002, 15:6-10.

58. Marchevsky AM, Wick MR: Evidence-based guidelines for the utilization of immunostains in diagnostic pathology: pulmonary adenocarcinoma versus mesothelioma. Appl Immunohistochem Mol Morphol 2007, 15:140-144.

59. Winters $\mathrm{NI}$, Thomason RT, Bader DM: Identification of a novel developmental mechanism in the generation of mesothelia. Development 2012, 139:2926-2934.

60. Rinkevich Y, Mori T, Sahoo D, Xu PX, Bermingham JR Jr, Weissman IL: Identification and prospective isolation of a mesothelial precursor lineage giving rise to smooth muscle cells and fibroblasts for mammalian internal organs, and their vasculature. Nat Cell Biol 2012, 14:1251-1260.

61. Tanaka M, Itoh T, Tanimizu N, Miyajima A: Liver stem/progenitor cells: their characteristics and regulatory mechanisms. J Biochem 2011, 149:231-239.

62. Herrick SE, Mutsaers SE: Mesothelial progenitor cells and their potential in tissue engineering. Int J Biochem Cell Biol 2004, 36:621-642.

doi:10.1186/2162-3619-3-12

Cite this article as: Zhang et al:: Expression and diagnostic values of calretinin and CK5/6 in cholangiocarcinoma. Experimental Hematology \& Oncology 2014 3:12

\section{Submit your next manuscript to BioMed Central and take full advantage of:}

- Convenient online submission

- Thorough peer review

- No space constraints or color figure charges

- Immediate publication on acceptance

- Inclusion in PubMed, CAS, Scopus and Google Scholar

- Research which is freely available for redistribution 